\title{
Excitation spectra of disordered dimer magnets near quantum criticality
}

\author{
Matthias Vojta \\ Institut für Theoretische Physik, Technische Universität Dresden, 01062 Dresden, Germany
}

(Dated: Aug 16, 2013)

\begin{abstract}
For coupled-dimer magnets with quenched disorder, we introduce a generalization of the bondoperator method, appropriate to describe both singlet and magnetically ordered phases. This allows for a numerical calculation of the magnetic excitations at all energies across the phase diagram, including the strongly inhomogeneous Griffiths regime near quantum criticality. We apply the method to the bilayer Heisenberg model with bond randomness and characterize both the broadening of excitations and the transfer of spectral weight induced by disorder. Inside the antiferromagnetic phase this model features the remarkable combination of sharp magnetic Bragg peaks and broad magnons, the latter arising from the tendency to localization of low-energy excitations.
\end{abstract}

Magnetic quantum phase transitions (QPT) have attracted enormous interest over the past two decades, with intriguing aspects such as Bose-Einstein condensation of magnons, exotic criticality, and non-Fermi liquid behavior 1 4]. The influence of quenched disorder, being inevitable in condensed-matter systems, on QPTs is a less explored field, although a number of theoretical results are available [5, [6]: Disorder can modify the critical behavior or even destroy the QPT; in addition it can produce singular response in off-critical systems via quantum Griffiths physics dominated by rare regions [7].

While the thermodynamics of disordered model systems near quantum criticality has been studied using a variety of theoretical tools [5], rather little is known about the dynamics of excitations in this fascinating regime, mainly because numerical methods are either restricted to the ground state or work in imaginary time where real-frequency spectra are difficult to extract, while analytical methods are restricted to low energy and to the limits of either weak or strong disorder. For quantum magnets, this issue is pressing, as high-resolution inelastic neutron scattering (INS) experiments are getting access to magnetic excitations near QPTs [8 11], and suitable materials with disorder created via intentional doping are available 12 15].

The aim of this paper is to close this gap: For coupleddimer magnets, being paradigmatic model systems for magnetic QPTs [1 3], we propose a generalization of the bond-operator method [16] to cases with quenched disorder. This enables the numerical calculation of magnetic excitation spectra in both paramagnetic and magnetically ordered phases, including the vicinity of the QPT, with disorder being treated exactly in finite-size systems. We apply the method to the square-lattice bilayer Heisenberg model, studied in detail in the disorder-free case 1722], with different types of exchange randomness. Near quantum criticality, we generically find a strong broadening in both energy and momentum of the low-energy response, while that at high energy is less affected by disorder. An interesting dichotomy follows upon slightly moving into the antiferromagnetic $(\mathrm{AF})$ phase: the strongly inhomogeneous AF features low-energy magnons that are distinctly broadened due to disorder-driven mode localization, but at the same time displays sharp Bragg peaks in its elastic response. We discuss connections to recent INS experiments.

Model. We consider a Heisenberg magnet of coupled pairs of spins $1 / 2$, with the general Hamiltonian

$$
\mathcal{H}=\sum_{i} J_{i} \vec{S}_{i 1} \cdot \vec{S}_{i 2}+\sum_{\left\langle i i^{\prime}\right\rangle m m^{\prime}} K_{i i^{\prime}}^{m m^{\prime}} \vec{S}_{i m} \cdot \vec{S}_{i^{\prime} m^{\prime}}
$$

defined on a lattice of $N$ dimer sites $i . J$ and $K$ are the intradimer and interdimer couplings, and $m=1,2$ labels the two spins of each dimer. Without quenched disorder in the couplings $J$ and $K$, this Hamiltonian can describe, e.g., spin ladders and bilayer Heisenberg magnets, but also applies to materials like $\mathrm{TlCuCl}_{3}[8,10,23,26]$ and $\mathrm{BaCuSi}_{2} \mathrm{O}_{6}$ 27 29].

Generalized bond-operator method. Let the four states of each dimer $i$ be $\left|t_{k}\right\rangle_{i}, k=0, \ldots, 3$, where $\left|t_{0}\right\rangle=(\mid \uparrow \downarrow$ \rangle$-|\downarrow \uparrow\rangle) / \sqrt{2},\left|t_{1}\right\rangle=(-|\uparrow \uparrow\rangle+|\downarrow \downarrow\rangle) / \sqrt{2},\left|t_{2}\right\rangle=i(\mid \uparrow \uparrow$ \rangle$+\downarrow \downarrow\rangle) / \sqrt{2},\left|t_{3}\right\rangle=(|\uparrow \downarrow\rangle+|\downarrow \uparrow\rangle) / \sqrt{2}$. Formally, bosonic operators $t_{i k}^{\dagger}$ can be introduced which create these states out of a fictituous vacuum, $\left|t_{k}\right\rangle_{i}=t_{i k}^{\dagger}|v a c\rangle_{i}$, with the constraint $\sum_{k} t_{i k}^{\dagger} t_{i k}=1$ defining the physical Hilbert space [16]. In a paramagnetic phase, it is convenient to fully "condense" the singlet, such that the operators $t_{i \alpha}^{\dagger}$ ( $\alpha=1,2,3)$ now create triplet excitations ("triplons") from a singlet background, and the constraint becomes of hard-core type, $\sum_{\alpha} t_{i \alpha}^{\dagger} t_{i \alpha} \leq 1$ [21]. Approximations can then be understood as expansion about the singlet product state $\left|\psi_{0}\right\rangle=\prod_{i}\left|t_{0}\right\rangle_{i}$.

Magnetically ordered phases correspond to a condensate of triplets, such that the reference state now involves a linear combination of singlet and triplets on each site. A consistent description of excitations requires a basis rotation in the four-dimensional Hilbert space spanned by the $\left|t_{k}\right\rangle[30,31]$. Here we generalize the approach of Ref. 30 to inhomogeneous states. For every dimer site, we introduce a $\mathrm{SU}(4)$ rotation to new basis states,

$$
\left|\tilde{t}_{k}\right\rangle_{i}=U_{k k^{\prime}}^{(i)}\left|t_{k^{\prime}}\right\rangle_{i}, \tilde{t}_{i k}^{\dagger}=U_{k k^{\prime}}^{(i)} t_{i k^{\prime}}^{\dagger} \quad\left(k, k^{\prime}=0, \ldots, 3\right)
$$

such that $\left|\tilde{\psi}_{0}\right\rangle=\prod_{i}\left|\tilde{t}_{0}\right\rangle_{i}$ replaces $\left|\psi_{0}\right\rangle$ as the reference 

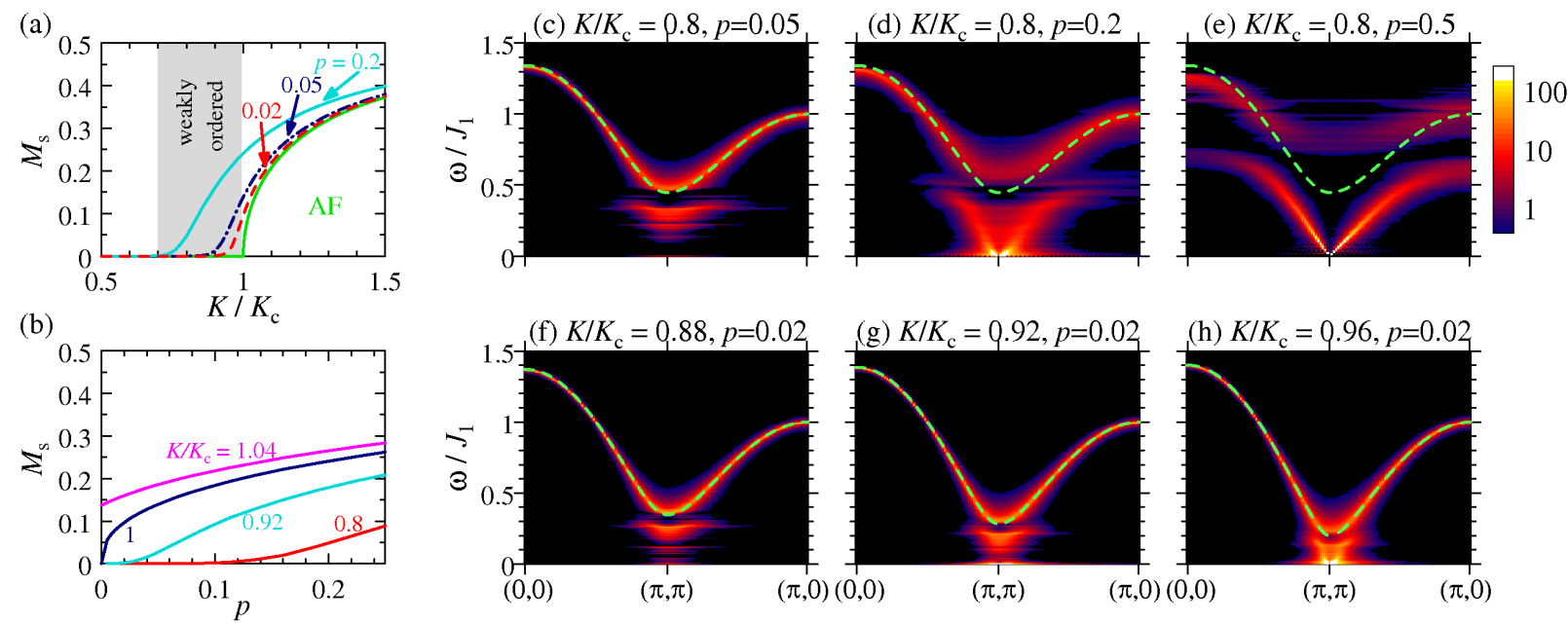

FIG. 1: (color online) Numerical results for the disordered bilayer Heisenberg model, with a concentration of $p$ weak interlayer couplings with $J_{2}=J_{1} / 2$. a) Phase diagram, showing the staggered magnetization $M_{s}$ as function of $K / K_{c}$ for different levels of disorder $p$, with $K_{c}=J_{1} / 4$ denoting the critical coupling of the clean system in the linearized bond-operator approach. A regime with weak and strongly inhomogeneous order emerges for small $p$ and $K \lesssim K_{c}$. b) $M_{s}$ as function of doping level $p$ for different $K$. c-h) Transverse dynamic susceptibility $\chi^{\prime \prime}(\vec{q}, \omega)$ along a path in the 2 d Brillouin zone $\left(q_{z}=\pi\right)$ for different combinations of $K / K_{c}$ and $p$. The green dashed lines indicate the dispersion of the clean system with the respective $K$.

state. For instance, $\left|\tilde{t}_{0}\right\rangle=\left(\left|t_{0}\right\rangle+\left|t_{3}\right\rangle\right) / \sqrt{2}=|\uparrow \downarrow\rangle$ describes a Néel state polarized along $z$. The $U^{(i)}$ are chosen such that $\left|\tilde{\psi}_{0}\right\rangle$ is the best product-state (i.e. saddlepoint) approximation to the ground state of $\mathcal{H}$ [32].

One now re-writes the Hamiltonian (1) in terms of the $\left|\tilde{t}_{k}\right\rangle_{i}$ using the transformation (2) 32]. In analogy to the paramagnetic case, one condenses $\tilde{t}_{0}^{\dagger}$, such that the $\tilde{t}_{\alpha}^{\dagger}(\alpha=1,2,3)$ describe excitations on top of the reference state $\left|\tilde{\psi}_{0}\right\rangle$. The Hamiltonian takes the form $\mathcal{H}=\mathcal{H}_{0}+\mathcal{H}_{1}+\mathcal{H}_{2}+\mathcal{H}_{3}+\mathcal{H}_{4}$, where $\mathcal{H}_{n}$ contains $n \tilde{t}_{\alpha}^{(\dagger)}$ operators, and an additional hard-core constraint for the $\tilde{t}_{\alpha}$ is implied. With the proper (saddle-point) choice of the reference state, $\mathcal{H}_{1}$ vanishes, and $\mathcal{H}_{2}$ describes Gaussian fluctuations around an inhomogeneous magnetic state. $\mathcal{H}_{2}$ has the form

$$
\mathcal{H}_{2}=\sum_{i j \alpha}\left[A_{i j} \tilde{t}_{i \alpha}^{\dagger} \tilde{t}_{j \alpha}+\left(\frac{B_{i j}}{2} \tilde{t}_{i \alpha} \tilde{t}_{j \alpha}+h . c .\right)\right]
$$

where quenched disorder enters via random $A_{i j}, B_{i j} . \mathcal{H}_{2}$ is solved by a bosonic Bogoliubov transformation, which yields the $3 N$ positive-energy eigenmodes of $\mathcal{H}_{2}$, used to calculate the dynamic spin susceptbility 32. In both paramagnetic and collinearly ordered phases, the polarization directions decouple, giving rise to triply degenerate modes in the paramagnetic case and a longitudinal and two transverse modes in the collinear case.

The excitations described by this method interpolate continuously between triplons of a paramagnet and spin waves of a semiclassical AF [30, 33]. The present harmonic approximation constitutes the leading-order correction to $\left|\tilde{\psi}_{0}\right\rangle$ in a systematic expansion in $1 / z$ with $z$ the number of neighbors [32, 34]. Anharmonic effects are neglected at this level; near criticality, this is qualitatively permissible if the anomalous exponent $\eta$ is small $[\eta=0.03$ for the Heisenberg model in $(2+1)$ dimensions]. Their quantitative effect on the dispersion can be captured via a renormalization of model parameters 35]; below we account for this by specifying parameters relative to the location of the QPT.

Bilayer magnet. In the remainder of the paper, we illustrate the application the method to a simple case, namely the bilayer Heisenberg model, as realized e.g. in $\mathrm{BaCuSi}_{2} \mathrm{O}_{6}$ 27 29]. Here, the dimers live on a $2 \mathrm{~d}$ square lattice with interlayer coupling $J$ and intralayer coupling $K=K_{i i^{\prime}}^{11}=K_{i i^{\prime}}^{22}$ for nearest-neighbor sites $i, i^{\prime}$. In the absence of disorder, this model is in a singlet ground state for $J \gg K$ and an AF ground state with ordering wavevector $\vec{Q}=(\pi, \pi)$ for $J \ll K$, with a $\mathrm{O}(3)$ critical point at $(J / K)_{c}=2.5220(1)$ 22]. In the harmonic bondoperator approach, this transition occurs at $(J / K)_{c}=4$; including triplon interactions allows one to obtain a value very close to the exact result [21].

With disorder of random-mass type, the character of the QPT changes, as dictated by the Harris criterion [36]. Numerical simulations have shown that a new critical point with conventional power-law singularities obtains 37] for not too strong disorder [38]. Here, we shall focus on the excitation spectrum in the vicinity of this QPT upon inclusion of disorder where signatures of quantum Griffiths behavior can be expected [5, 6].

Modelling disorder. We shall mainly employ bimodal distributions of coupling constants, being experimentally relevant to cases where chemical substitution modifies exchange paths, as occurs, e.g., in $\mathrm{Tl}_{1-x} \mathrm{~K}_{x} \mathrm{CuCl}_{3}$ [39] or $\left(\mathrm{C}_{4} \mathrm{H}_{12} \mathrm{~N}_{2}\right) \mathrm{Cu}_{2}\left(\mathrm{Cl}_{1-x} \mathrm{Br}_{x}\right)_{6}$ [14]. Provided that all cou- 
plings remain AF, this type of bond randomness does not introduce frustration, such that the magnetic order realized for $J \ll K$ continues to be collinear with wavevector $\vec{Q}=(\pi, \pi)$. We will denote the corresponding order parameter, the staggered magnetization per spin, by $M_{s}$.

Results: Weak intradimer bonds and evolution across QPT. We now present numerical results of our approach, for disordered intradimer coupling $J$, with values $J_{1}$ and $J_{2}$ taken with probabilities $(1-p)$ and $p$, respectively, and use the interdimer coupling $K$ as a tuning parameter to access the transition. Energies will be quoted in units of $J_{1}$, the system size is $N=L^{2}$ with $L=64$ and additional $2^{2}$ supercells [32], disorder averages are performed over $N_{\mathrm{r}}=50$ realizations, and the temperature is $T=0$.

Results for small concentrations $p$ of weak intradimer bonds, $J_{2}=J_{1} / 2$, are in Fig. 11 Panels (a,b) display $M_{s}$ as function of $K$ and $p$, while panels (c)-(h) show the dynamical susceptibility $\chi^{\prime \prime}(\vec{q}, \omega)$ for different parameter sets. This type of substitution drives the system towards the ordered phase, by decreasing the apparent gap and shifting the QPT to smaller $K$ upon increasing $p$. There is a broad range of parameters with weak magnetic order, i.e., small non-zero $M_{s}$, to be discussed below.

Introducing a small amount $p$ of disorder into the paramagnetic clean system causes a transfer of spectral weight to low energies, Fig. 1 (c,f-h) and Fig. 2 Doping creates excitations inside the gap which eventually induce weak order upon increasing $p$. This disorder-induced lowenergy response, although centered around the ordering wavevector $\vec{Q}$, is broad in both momentum and energy (except for Goldstone modes at extremely small $\omega$ ): This reflects the localization tendency of the corresponding modes, see Fig. 3. For intermediate $p$ near $1 / 2$, the spectrum visibly separates into upper and lower branches which exist over the entire Brillouin zone, Fig. 1(e); these branches correspond to modes which are primarily carried by either the $J_{1}$ or the $J_{2}$ intradimer bonds.

We now turn to a detailed analysis of the weak substitution-induced magnetic order. Fig. 3 portraits a single realization of disorder, by showing the spatial distribution of $J$ and the local magnetization $M_{i}$ as well as
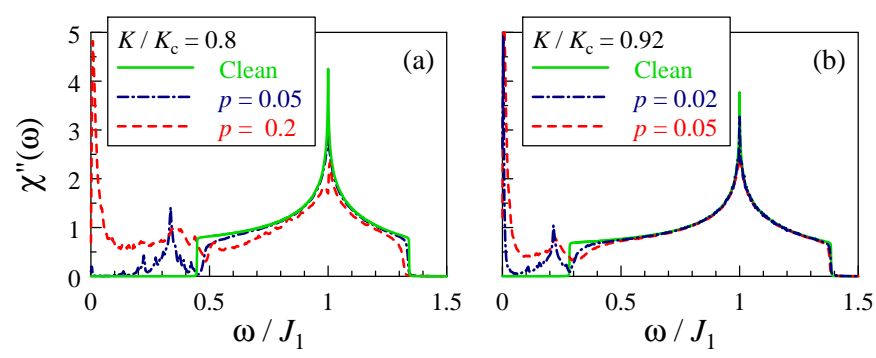

FIG. 2: (color online) Momentum-integrated transverse susceptibility $\chi^{\prime \prime}(\omega)$, for the bilayer model with random weak interlayer couplings as in Fig. 1 Disorder tends to close the spin gap by transferring of spectral weight to low energies.
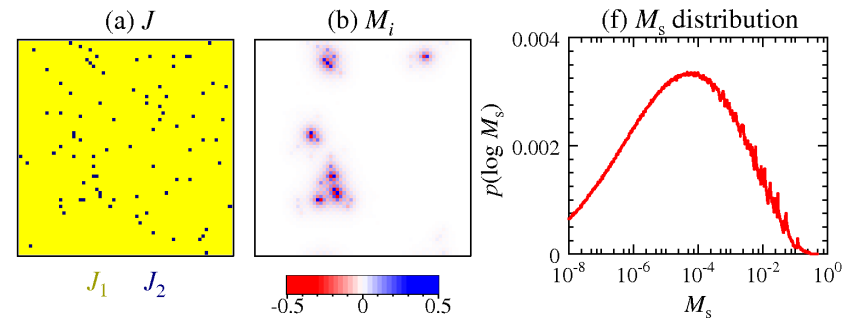

(c) $\omega / J_{1}=0.126$
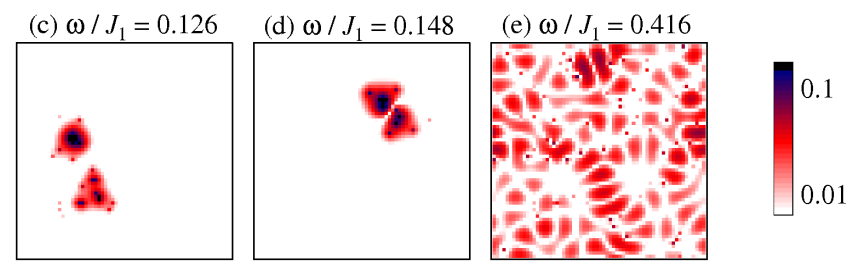

FIG. 3: (color online) Bilayer Heisenberg magnet with $K / K_{c}=0.92$ and a single disorder realization of $p=0.02$ weak intradimer bonds with $J_{2}=J_{1} / 2$, as in Fig. 1 (g), with $L=64$. (a) Spatial distribution of $J$ values, indicating the location of the weak bonds. (b) Corresponding spatial distribution of the local magnetization $M_{i}$. (c)-(e) Wavefunction amplitudes of selected eigenmodes of $\mathcal{H}_{2}$. (f) Disorderaveraged probability distribution of local (staggered) magnetization values for parameters as in (a)-(e).

the wavefunctions for selected eigenmodes of $\mathcal{H}_{2}$. Several features are apparent: (i) The system develops islands of non-zero staggered magnetization, Fig. 3.(b), with a characteristic length scale of $\xi$, the correlation length of the clean reference system. These islands, being rare events in the sense of Griffiths 7], exist in regions with larger concentration of weak interlayer bonds. (ii) The distribution of local magnetization values becomes broad on logarithmic scales, Fig. 3(f), a typical fingerprint of Griffiths behavior. (iii) The low-energy "magnon" modes appear strongly localized on individual magnetization islands, Fig. 3( c,d). This behavior persists for essentially all energies below the gap of the clean reference system, whereas higher-energy modes tend to be delocalized, Fig. 3(e). We have confirmed this localization tendency by analyzing the inverse participation ratio [32].
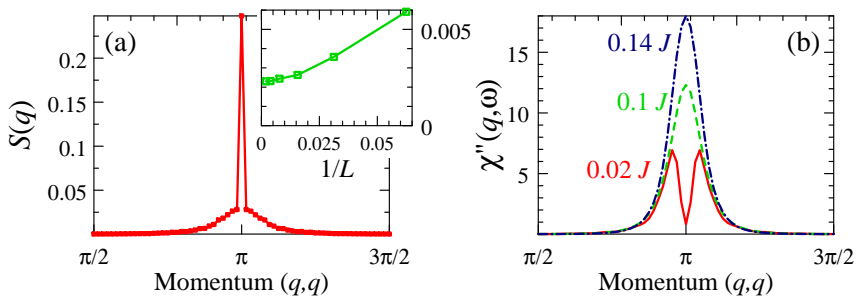

FIG. 4: (color online) (a) Static structure factor $S(\vec{q})$ and (b) susceptibility $\chi^{\prime \prime}(\vec{q}, \omega)$ at different $\omega$, for $K / K_{c}=0.92$ and $p=0.02$ weak intradimer bonds for $L=128$. The inset in (a) shows the finite-size scaling of $[S(\vec{Q}) /(2 N)]^{1 / 2}$ which equals the order parameter $M_{s}$ in the thermodynamic limit. 
(a) $K / K_{\mathrm{c}}=0.96, p=0.1$

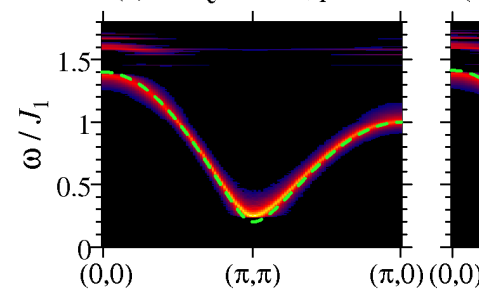

(b) $K / K_{\mathrm{c}}=1.0, p=0.1$

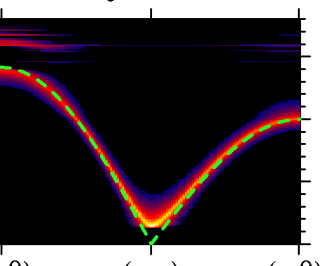

(c) $K / K_{\mathrm{c}}=1.02, p=0.1$

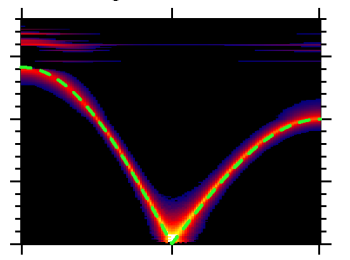

$(\pi, \pi)$ (d) $K / K_{\mathrm{c}}=1.04, p=0.1$

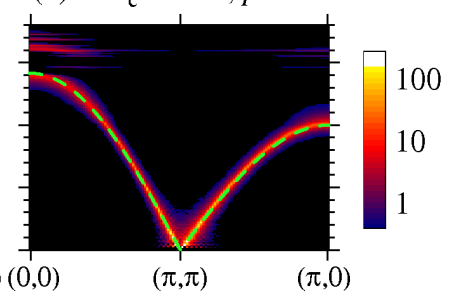

FIG. 5: Results for the susceptibility $\chi^{\prime \prime}(\vec{q}, \omega)$ as in Fig. 1 but for a density of $p$ strong intradimer couplings with $J_{2}=3 J_{1} / 2$.

Taken together, this implies an interesting "dual" nature of the weakly ordered phase: It displays a sharp Bragg peak in the static structure factor $S(\vec{q})$ at $\vec{Q}=$ $(\pi, \pi)$, Fig. 4(a), as the underlying order is perfectly staggered (albeit strongly inhomogeneous). At the same time, the small- $\omega$ dynamic response $\chi^{\prime \prime}(\vec{q}, \omega)$ is anomalously broad in $q$-space, due to the disorder-induced mode localization. This is shown in Fig. 4(b), where a spinwave-like two-peak structure is only visible for extremely small $\omega / J \lesssim 0.02$.

Related localization and broadening phenomena have been observed and discussed both theoretically and experimentally for a variety of random magnets, mainly inside magnetically ordered [40 46] or quantum-disordered phases [47, 48]. Our results show that, due to Griffiths physics, low-energy localization tendencies are significantly stronger near quantum criticality than deep inside stable phases; for an extended discussion see Ref. 32 .

Further results. We have studied other types of disorder, with selected results in Fig. 5 and the supplemental material [32]. Depending on the type of disorder, the overall triplon bandwidth may either increase or decrease with doping; the same applies to the apparent gap in $\chi^{\prime \prime}(\omega)$. For example, few strong intradimer bonds decrease the width of main band, Fig. 15 because coherent triplon hopping is preempted through the strong bonds. Band splitting like in Fig. 1(e) is visible for sizeable bimodal disorder; for continuous distributions this is replaced by strong smearing of intensity. Most importantly, spectral broadening at low energies appears generic near the QPT; this effect is strongest if the dopants tend to drive the system towards the ordered phase, as in Fig. 1.

We have also performed calculations for other $2 \mathrm{~d}$ unfrustrated coupled-dimer models, with qualitatively similar results which thus appear generic.

Comparison to experiments. Recent experiments 12, 13, 15] have studied bond randomness by ligand doping in spin-ladder materials. INS shows excitations with a larger energy width and an increased spin gap as compared to the undoped case. This consistent with our results for dopants which create locally stronger intradimer couplings, as in Fig. 5, or weaker interdimer couplings or both. For the investigated materials, such properties can indeed be deduced from studies of the end members of the doping series [32].

While INS data of bond-disordered nearly-critical dimer magnets in $2 \mathrm{~d}$ or $3 \mathrm{~d}$ are not available to our knowledge, it is interesting to connect our results to $\mathrm{La}_{2-x} \mathrm{Sr}_{x} \mathrm{CuO}_{4}$ : At $x=0.145$ this compound displays a field-driven magnetic ordering transition, where INS data indicate the closing of a spin gap, but in the presence of a non-diverging correlation length [11]. Since it is known that $\mathrm{La}_{2}-x \mathrm{Sr}_{x} \mathrm{CuO}_{4}$ shows spatially disordered charge stripes [49] which in turn lead to modulated magnetic couplings, our modelling of random-mass disorder can be expected to qualitatively describe the soft-mode behavior of $\mathrm{La}_{2-x} \mathrm{Sr}_{x} \mathrm{CuO}_{4}$. Therefore we propose that quenched disorder tends to localize low-energy magnetic modes at the QPT in $\mathrm{La}_{2-x} \mathrm{Sr}_{x} \mathrm{CuO}_{4}$, thus providing a cutoff to the apparent magnetic correlation length. Disorder is also expected to cut-off one-parameter scaling in $\chi^{\prime \prime}(\omega, T)$ - this is testable in future experiments.

Summary. For coupled-dimer magnets, we have proposed an efficient method to calculate real-frequency excitation spectra in the presence bond randomness across the entire phase diagram. This allows one in particular to study magnetic excitations near quantum criticality, where the effect of disorder is generically strong. Using the bilayer Heisenberg model as an example, we have studied disorder-induced spectral weight transfer and weak magnetic order. We find strong broadening of low-energy excitation spectra due the localization tendency of the relevant modes. Further high-resolution INS experiments, e.g., on $\mathrm{Tl}_{1-x} \mathrm{~K}_{x} \mathrm{CuCl}_{3}$ [39], which could test our predictions are called for.

Our method can be extended to the case with magnetic field, in order to access excitations of disordered magnon Bose condensates, Bose glasses, or disordered supersolids. A generalization to frustrated dimer lattices and to incommensurate order is possible as well.

I thank E. Andrade, S. Burdin, J. Chang, R. Doretto, D. Joshi, M. Müller, C. Rüegg, and S. Wessel for discussions. This research was supported by the DFG through FOR 960 and GRK 1621. In addition, financial support by the Heinrich-Hertz-Stiftung NRW and the hospitality of the Centro Atomico Bariloche during early stages of this project are gratefully acknowledged. 
[1] S. Sachdev, Quantum Phase Transitions, 2nd ed., Cambridge University Press, Cambridge (2010).

[2] S. Sachdev, Nature Phys. 4, 173 (2008).

[3] T. Giamarchi, C. Rüegg, and O. Tchernyshyov, Nature Phys. 4, 198 (2008).

[4] H. von Löhneysen, A. Rosch, M. Vojta, and P. Wölfle, Rev. Mod. Phys. 79, 1015 (2007).

[5] T. Vojta, J. Phys. A 39, R143 (2006), and references therein.

[6] T. Vojta, J. Low Temp. Phys. 161, 299 (2010).

[7] R. B. Griffiths, Phys. Rev. Lett. 23, 17 (1969).

[8] C. Rüegg, A. Furrer, D. Sheptyakov, T. Strässle, K. W. Krämer, H.-U. Güdel, and L. Melesi, Phys. Rev. Lett. 93, 257201 (2004).

[9] B. Lake, D. A. Tennant, C. D. Frost, and S. E. Nagler, Nature Mat. 4, 329 (2005).

[10] C. Rüegg, B. Normand, M. Matsumoto, A. Furrer, D. F. McMorrow, K. W. Krämer, H.-U. Güdel, S. Gvasaliya, H. Mutka, and M. Boehm, Phys. Rev. Lett. 100, 205701 (2008).

[11] J. Chang, N. B. Christensen, C. Niedermayer, K. Lefmann, H. M. Rønnow, D. F. McMorrow, A. Schneidewind, P. Link, A. Hiess, M. Boehm, R. Mottl, S. Pailhes, N. Momono, M. Oda, M. Ido, and J. Mesot, Phys. Rev. Lett. 102, 177006 (2009).

[12] M. B. Stone, A. Podlesnyak, G. Ehlers, A. Huq, E. C. Samulon, M. C. Shapiro, and I. R. Fisher, J. Phys. Cond. Matter 23, 416003 (2011).

[13] D. Hüvonen, S. Zhao, M. Månsson, T. Yankova, E. Ressouche, C. Niedermayer, M. Laver, S. N. Gvasaliya, and A. Zheludev, Phys. Rev. B 85, 100410 (2012).

[14] D. Hüvonen, S. Zhao, G. Ehlers, M. Månsson, S. N. Gvasaliya, and A. Zheludev, Phys. Rev. B 86, 214408 (2012).

[15] B. Nafradi, T. Keller, H. Manaka, U. Stuhr, A. Zheludev, and B. Keimer, Phys. Rev. B 87, 020408(R) (2013).

[16] S. Sachdev and R. N. Bhatt, Phys. Rev. B 41, 9323 (1990).

[17] T. Matsuda and K. Hida, J. Phys. Soc. Jpn. 59, 2223 (1990); K. Hida, ibid., 2230 (1990).

[18] K. Hida, J. Phys. Soc. Jpn. 61, 1013 (1992).

[19] A. W. Sandvik and D. J. Scalapino, Phys. Rev. Lett. 72, 2777 (1994).

[20] A. V. Chubukov and D. K. Morr, Phys. Rev. B 52, 3521 (1995).

[21] V. N. Kotov, O. P. Sushkov, Zheng Weihong, and J. Oitmaa, Phys. Rev. Lett. 80, 5790 (1998).

[22] L. Wang, K. S. D. Beach, and A. W. Sandvik, Phys. Rev. B 73, 014431 (2006).

[23] K. Takatsu, W. Shiramura, and H. Tanaka, J. Phys. Soc. Jpn. 66, 1611 (1997).

[24] A. Oosawa, M. Ishii, and H. Tanaka, J. Phys.: Condens. Matter 11, 265 (1999).
[25] Ch. Rüegg, N. Cavadini, A. Furrer, H.-U. Güdel, K. Krämer, H. Mutka, A. Wildes, K. Habicht, and P. Vorderwisch, Nature 423, 62 (2003).

[26] M. Matsumoto, B. Normand, T. M. Rice, and M. Sigrist, Phys. Rev. Lett. 89, 077203 (2002).

[27] Y. Sasago, K. Uchinokura, A. Zheludev, and G. Shirane, Phys. Rev. B 55, 8357 (1997).

[28] M. Jaime, V. F. Correa, N. Harrison, C. D. Batista, N. Kawashima, Y. Kazuma, G. A. Jorge, R. Stern, I. Heinmaa, S. A. Zvyagin, Y. Sasago, and K. Uchinokura, Phys. Rev. Lett. 93, 087203 (2004).

[29] S. E. Sebastian, N. Harrison, C. D. Batista, L. Balicas, M. Jaime, P. A. Sharma, N. Kawashima, and I. R. Fisher, Nature 441, 617 (2006).

[30] T. Sommer, M. Vojta, and K. W. Becker, Eur. Phys. J. B 23, 329 (2001).

[31] An approach similar to Ref. 30 has been developed independently in: J. Romhanyi, K. Totsuka, and K. Penc, Phys. Rev. B 83, 024413 (2011).

[32] See Supplemental Material for a description of our numerical approach, additional results for the spectra and localization properties, and a brief discussion of previous work on excitations in disordered magnets.

[33] M. Vojta and T. Ulbricht, Phys. Rev. Lett. 93, 127002 (2004).

[34] D. Joshi and M. Vojta, in preparation.

[35] R. Eder, Phys. Rev. B 57, 12832 (1998).

[36] A. B. Harris, J. Phys. C 7, 1671 (1974).

[37] R. Sknepnek, T. Vojta, and M. Vojta, Phys. Rev. Lett. 93, 097201 (2004).

[38] Y.-C. Lin, H. Rieger, N. Laflorencie, and F. Igloi, Phys. Rev. B 74, 024427 (2006).

[39] A. Oosawa and H. Tanaka, Phys. Rev. B 65, 184437 (2002).

[40] R. A. Cowley and W. J. L. Buyers, Rev. Mod. Phys. 44, 406 (1972).

[41] S. E. Nagler, W. J. L. Buyers, R. L. Armstrong, and R. A. Ritchie, J. Phys. C 17, 4819 (1984).

[42] Y. J. Uemura and R. J. Birgeneau, Phys. Rev. B 36, 7024 (1987).

[43] A. L. Chernyshev, Y. C. Chen, and A. H. Castro-Neto, Phys. Rev. B 65, 104407 (2002).

[44] E. R. Mucciolo, A. H. Castro Neto, and C. Chamon, Phys. Rev. B 69, 214424 (2004).

[45] A. Chakraborty and G. Bouzerar, Phys. Rev. B 81, 172406 (2010).

[46] A. L. Chernyshev, M. E. Zhitomirsky, N. Martin, and L.-P. Regnault, Phys. Rev. Lett. 109, 097201 (2012).

[47] G. Xu, G. Aeppli, M. E. Bisher, C. Broholm, J. F. DiTusa, C. D. Frost, T. Ito, K. Oka, R. L. Paul, H. Takagi, and M. M. J. Treacy, Science 289, 419 (2000).

[48] S. Haravifard, S. R. Dunsiger, S. El Shawish, B. D. Gaulin, H. A. Dabkowska, M. T. F. Telling, T. G. Perring, and J. Bonca, Phys. Rev. Lett. 97, 247206 (2006).

[49] M. Vojta, Adv. Phys. 58, 699 (2009). 


\title{
Supplementary information for: Excitation spectra of disordered dimer magnets near quantum criticality
}

\author{
Matthias Vojta \\ Institut für Theoretische Physik, Technische Universität Dresden, 01062 Dresden, Germany
}

(Dated: Aug 16, 2013)

\section{BOND OPERATORS WITH DISORDER: NUMERICS}

In the following we describe details of our bondoperator approach to dimer magnets with quenched disorder, in particular the numerical implementation. We work with finite-size systems of $N=L^{2}$ dimers on a square lattice with periodic boundary conditions; the generalization to other lattices is straightforward.

\section{A. Optimum product state}

The basis rotation in the four-dimensional Hilbert space of each dimer, Eq. (2) in the main text, is determined by the requirement that $\left|\tilde{\psi}_{0}\right\rangle=\prod_{i}\left|\tilde{t}_{0}\right\rangle_{i}$ is the best product-state approximation to the ground state of $\mathcal{H}$. The state $\left|\tilde{\psi}_{0}\right\rangle$ can be obtained in various ways: (i) by minimizing $\mathcal{H}_{0}=\left\langle\tilde{\psi}_{0}|\mathcal{H}| \tilde{\psi}_{0}\right\rangle$, (ii) by demanding $\mathcal{H}_{1}$ to vanish, as the latter would create a condensate of $\tilde{t}_{\alpha}$ bosons, and (iii) dimer mean-field theory. It is easy to see that these methods are equivalent: First, upon minimizing $\mathcal{H}_{0}$ one finds a saddle point where by construction linear variations (and thus $\mathcal{H}_{1}$ ) vanish. Second, the minimum condition can be converted into mean-field theory as follows. The variation of $\left\langle\tilde{\psi}_{0}|\mathcal{H}| \tilde{\psi}_{0}\right\rangle$ w.r.t. $\left|\tilde{t}_{0}\right\rangle_{i}$ or, equivalently w.r.t. $U_{0 k^{\prime}}^{(i)}$ reads

$$
\frac{\delta\langle\mathcal{H}\rangle}{\delta U_{0 k^{\prime}}^{(i)}}=J_{i} \frac{\delta\left\langle\vec{S}_{i 1} \cdot \vec{S}_{i 2}\right\rangle_{i}}{\delta U_{0 k^{\prime}}^{(i)}}+\sum_{m} \frac{\delta\left\langle\vec{S}_{i m}\right\rangle_{i}}{\delta U_{0 k^{\prime}}^{(i)}} \cdot \vec{h}_{i m}=0
$$

where $\langle\ldots\rangle_{i}$ is a shorthand for ${ }_{i}\left\langle\tilde{t}_{0}|\ldots| \tilde{t}_{0}\right\rangle_{i}$ and $\vec{h}_{i m}=$ $\sum_{i^{\prime} m^{\prime}} K_{i i^{\prime}}^{m m^{\prime}}\left\langle\vec{S}_{i^{\prime} m^{\prime}}\right\rangle_{i^{\prime}}$. The condition Eq. (S1) is the same as the minimum condition for the single-dimer mean-field Hamiltonian

$$
\mathcal{H}_{\mathrm{MF}}^{(i)}=J_{i} \vec{S}_{i 1} \cdot \vec{S}_{i 2}+\sum_{i^{\prime} m m^{\prime}} K_{i i^{\prime}}^{m m^{\prime}} \vec{S}_{i m} \cdot\left\langle\vec{S}_{i^{\prime} m^{\prime}}\right\rangle_{i^{\prime}} .
$$

For this single-dimer problem the variation w.r.t. $\left|\tilde{t}_{0}\right\rangle_{i}$ is a variation in the full Hilbert space and hence yields the exact ground state. This establishes the equivalence of the three criteria. We also note that $\left|\tilde{\psi}_{0}\right\rangle$ becomes the exact ground state of $\mathcal{H}$ in the limit of infinite coordination number between the dimers, see Sec. IIB below.

To practically determine the optimum collinearly ordered product state for the Hamiltonian $\mathcal{H}$ with a given realization of disorder, i.e. given random couplings, we have found it most efficient to iterate the coupled set of
$N$ mean-field problems in Eq. (S2) until convergence: We start with an initial collinear guess for the $\left\langle\vec{S}_{i m}\right\rangle$. Then, diagonalizing the $4 \times 4$ Hamiltonian $\mathcal{H}_{\mathrm{MF}}^{(i)}$ in Eq. (S2) for each dimer site $i$ gives a $4 \times 4$ matrix of eigenvectors which we choose to be $U^{(i)}$. In particular, the lowest-energy eigenvector $U_{0 k^{\prime}}^{(i)}$ can be used to calculate new $T=0$ values of the $\left\langle\vec{S}_{i m}\right\rangle$, which closes the self-consistency loop.

\section{B. Conversion of the Hamiltonian}

To calculate the excitation spectrum, the Hamiltonian $\mathcal{H}$ needs to be expressed in terms of the local $\tilde{t}_{i \alpha}$ excitation operators, as described in the following.

The starting point is the representation of the spins $\vec{S}_{i m}$ via transition operators between the states $\left|t_{k}\right\rangle_{i}$ of a dimer,

$$
S_{i m}^{\alpha}=\sum_{k k^{\prime}} s_{k k^{\prime}}^{\alpha m}\left|t_{k}\right\rangle_{i i}\left\langle t_{k^{\prime}}\right|
$$

with $4 \times 4$ matrices $s^{\alpha m}$ for the spin components $S^{\alpha}(\alpha=$ $x, y, z \equiv 1,2,3)$ of the $m=1,2$ spins:

$$
\begin{aligned}
& s^{x 1,2}=\frac{1}{2}\left(\begin{array}{cccc}
0 & \pm 1 & 0 & 0 \\
\pm 1 & 0 & 0 & 0 \\
0 & 0 & 0 & -i \\
0 & 0 & i & 0
\end{array}\right), \\
& s^{y 1,2}=\frac{1}{2}\left(\begin{array}{cccc}
0 & 0 & \pm 1 & 0 \\
0 & 0 & 0 & i \\
\pm 1 & 0 & 0 & 0 \\
0 & -i & 0 & 0
\end{array}\right), \\
& s^{z 1,2}=\frac{1}{2}\left(\begin{array}{cccc}
0 & 0 & 0 & \pm 1 \\
0 & 0 & -i & 0 \\
0 & i & 0 & 0 \\
\pm 1 & 0 & 0 & 0
\end{array}\right) .
\end{aligned}
$$

This is of course equivalent to the bond-operator representation of Sachdev and Bhatt,,

$$
S_{i 1,2}^{\alpha}=\frac{1}{2}\left( \pm t_{i 0}^{\dagger} t_{i \alpha} \pm t_{i \alpha}^{\dagger} t_{i 0}-i \epsilon_{\alpha \beta \gamma} t_{i \beta}^{\dagger} t_{i \gamma}\right),
$$

in the Hilbert space with $\sum_{k} t_{i k}^{\dagger} t_{i k}=1$ bosons. After the basis rotation (2), Eq. (S3) becomes

$$
S_{i m}^{\alpha}=\sum_{k k^{\prime}} \tilde{s}_{i, k k^{\prime}}^{\alpha m}\left|\tilde{t}_{k}\right\rangle_{i} i\left\langle\tilde{t}_{k^{\prime}}\right|
$$


with the transformed spin matrices now being sitedependent:

$$
\tilde{s}_{i, k k^{\prime}}^{\alpha m}=\sum_{l l^{\prime}}\left(U^{\dagger}\right)_{l k}^{(i)} s_{l l^{\prime}}^{\alpha m} U_{k^{\prime} l^{\prime}}^{(i)}
$$

The full Hamiltonian in terms of transition operators takes the (exact) form

$$
\begin{aligned}
\mathcal{H} & =\sum_{i k k^{\prime}} X_{i}^{k k^{\prime}}\left|\tilde{t}_{k}\right\rangle_{i}{ }_{i}\left\langle\tilde{t}_{k^{\prime}}\right| \\
& +\sum_{i i^{\prime} k k^{\prime} l l^{\prime}} Y_{i i^{\prime}}^{k k^{\prime} l l^{\prime}}\left|\tilde{t}_{k}\right\rangle_{i}\left|\tilde{t}_{l}\right\rangle_{i^{\prime}}{ }_{i}\left\langle\left.\tilde{t}_{k^{\prime}}\right|_{i^{\prime}}\left\langle\tilde{t}_{l^{\prime}}\right| .\right.
\end{aligned}
$$

Its coefficients $X_{i}^{k k^{\prime}}$ and $Y_{i i^{\prime}}^{k k^{\prime} l l^{\prime}}$ are related to the original couplings $J_{i}$ and $K_{i i^{\prime}}^{m m^{\prime}}$, respectively. They can be generated numerically for arbitrary $U_{k k^{\prime}}^{(i)}$ of a finite-size system with a given realization of disorder, using the equations (1), (S6), and (S7).

This Hamiltonian is now rewritten into $\tilde{t}_{i \alpha}(\alpha=1,2,3)$ bosonic operators which are defined to act on the background state $\prod_{i}\left|\tilde{t}_{0}\right\rangle_{i}$, i.e., $\tilde{t}_{i \alpha}^{\dagger}\left|\tilde{t}_{0}\right\rangle_{i}=\left|\tilde{t}_{\alpha}\right\rangle_{i}$. Using the decomposition $\mathcal{H}=\mathcal{H}_{0}+\mathcal{H}_{1}+\mathcal{H}_{2}+\mathcal{H}_{3}+\mathcal{H}_{4}$ we have

$$
\begin{aligned}
\mathcal{H}_{0} & =\sum_{i} X_{i}^{00}+\sum_{i i^{\prime}} Y_{i i^{\prime}}^{0000}, \\
\mathcal{H}_{1} & =\sum_{i \alpha}\left(X_{i}^{0 \alpha} \tilde{t}_{i \alpha}+X_{i}^{\alpha 0} \tilde{t}_{i \alpha}^{\dagger}\right)+\sum_{i i^{\prime} \alpha}\left(Y_{i i^{\prime}}^{0 \alpha 00} \tilde{t}_{i \alpha}+Y_{i i^{\prime}}^{000 \alpha} \tilde{t}_{i^{\prime} \alpha}+Y_{i i^{\prime}}^{\alpha 000} \tilde{t}_{i \alpha}^{\dagger}+Y_{i i^{\prime}}^{00 \alpha 0} \tilde{t}_{i^{\prime} \alpha}^{\dagger}\right), \\
\mathcal{H}_{2} & =\sum_{i \alpha \beta}\left(X_{i}^{\alpha \beta}-X_{i}^{00} \delta_{\alpha \beta}\right) \tilde{t}_{i \alpha}^{\dagger} \tilde{t}_{i \beta}+\sum_{i i^{\prime} \alpha \beta}\left[\left(Y_{i i^{\prime}}^{\alpha \beta 00}-Y_{i i^{\prime}}^{0000} \delta_{\alpha \beta}\right) \tilde{t}_{i \alpha}^{\dagger} \tilde{t}_{i \beta}\left(Y_{i i^{\prime}}^{00 \alpha \beta}-Y_{i i^{\prime}}^{0000} \delta_{\alpha \beta}\right) \tilde{t}_{i^{\prime} \alpha}^{\dagger} \tilde{t}_{i^{\prime} \beta}\right] \\
& +\sum_{i i^{\prime} \alpha \beta}\left(Y_{i i^{\prime}}^{\alpha 00 \beta} \tilde{t}_{i \alpha}^{\dagger} \tilde{t}_{i^{\prime} \beta}+Y_{i i^{\prime}}^{0 \beta \alpha 0} \tilde{t}_{i^{\prime} \alpha}^{\dagger} \tilde{t}_{i \beta}+Y_{i i^{\prime}}^{\alpha 0 \beta 0} \tilde{t}_{i \alpha}^{\dagger} \tilde{t}_{i^{\prime} \beta}^{\dagger}+Y_{i i^{\prime}}^{0 \alpha 0 \beta} \tilde{t}_{i \alpha} \tilde{t}_{i^{\prime} \beta}\right)
\end{aligned}
$$

where $\alpha, \beta=1,2,3$. Similar expressions can be found for $\mathcal{H}_{3}$ and $\mathcal{H}_{4}$ which are, however, not needed in the following. In each run, we have checked that $\mathcal{H}_{1}$ vanishes to numerical accuracy provided that the iteration of the product state has converged.

\section{Gaussian fluctuations and susceptibility}

The Gaussian fluctuations of interest are contained in $\mathcal{H}_{2}$, which describes $3 N$ modes where $N$ is the number of dimers, and the factor of three arises from the spin polarizations $\alpha=x, y, z$.

We generate a matrix form of $\mathcal{H}_{2}$ numerically from the coefficients $X_{i}^{k k^{\prime}}$ and $Y_{i i^{\prime}}^{k k^{\prime} l l^{\prime}}$ via Eq. (S9). In both the paramagnetic and the collinearly ordered cases studied here, the polarization directions decouple, such that $\mathcal{H}_{2}$ is block-diagonal. Then, it remains to perform a bosonic Bogoliubov transformation for the transverse and longitudinal fluctuations separately. Each amounts to the numerical diagonalization of a $2 N \times 2 N$ non-Hermitean matrix. We have implemented the procedure described in the appendix of Ref. 2 using standard LAPACK routines. An efficient implementation can be achieved using the OpenMP version of Intel's MKL library, which allowed us to access system sizes up to $N=128^{2}$ in the collinear case using a desktop computer.

Each diagonalization yields (per polarization) $2 N$ eigenvectors and eigenvalues, with the latter coming in pairs with opposite sign. In the presence of a symmetrybreaking condensate, we always find at least one pair of eigenvalues with magnitude smaller than $10^{-5} J$ in the transverse piece of $\mathcal{H}_{2}$, corresponding to the expected zero-energy (Goldstone) mode.

The positive-energy eigenvalues $\omega_{n}$ and their eigenvectors $\left(u_{n}, v_{n}\right)$ describe the Gaussian fluctuation modes $\tau_{n}$ of $\mathcal{H}$, which are linear combinations of the $\tilde{t}$ operators:

$$
\tau_{n}=\sum_{i \alpha}\left(u_{n i \alpha} \tilde{t}_{i \alpha}+v_{n i \alpha} \tilde{t}_{i \alpha}^{\dagger}\right) .
$$

The $\omega_{n}$ and $\left(u_{n}, v_{n}\right)$ can be used to extract physical observables. In the paper, we have concentrated on the dynamic spin susceptbility at $T=0$ in the single-magnon approximation

$$
\chi_{\alpha}^{\prime \prime}(\vec{q}, \omega)=\left[\sum_{n}\left|M_{n}^{\alpha}(\vec{q})\right|^{2} \delta\left(\omega-\omega_{n}\right)\right]_{\mathrm{av}}
$$

where []$_{\text {av }}$ denotes disorder averaging, and $M_{n}^{\alpha}(\vec{q})$ is the matrix element $\left\langle 0\left|S^{\alpha}(\vec{q})\right| n\right\rangle$ of the Fourier-transformed spin operator (in the single-magnon approximation),

$$
S^{\alpha}(\vec{q})=\sum_{i m} e^{i \vec{q} \cdot \vec{r}_{i m}} \sum_{\beta}\left(\tilde{s}_{i \beta 0}^{\alpha m} \tilde{t}_{i \beta}^{\dagger}+\tilde{s}_{i 0 \beta}^{\alpha m} \tilde{t}_{i \beta}\right)
$$

with the eigenmode $|n\rangle=\tau_{n}^{\dagger}|0\rangle$. Here, $\vec{r}_{i m}$ is the lattice coordinate of an individual spin. For the bilayer model under consideration, $\vec{q}$ has a $z$ component $q_{z}=0, \pi$; we 
restrict our attention to the staggered response, $q_{z}=$ $\pi$, because the low-energy modes have significant weight only in this channel.

For a $L \times L$ system, $\chi_{\alpha}^{\prime \prime}(\vec{q}, \omega)$ can then be calculated with a momentum resolution in $\left(q_{x}, q_{y}\right)$ of $L$ points per direction. The delta function in Eq. (S11) may be converted into a Lorentzian with artificial width. However, for momentum-resolved spectra we found it advantageous to use binning instead, because the exact intensity in the clean case diverges as $1 / \omega$ upon approaching $\vec{Q}$ for transverse (Goldstone) modes, such that a Lorentzian broadening leads to unphysical weight at elevated energies.

In order to improve the momentum resolution, we have also implemented twisted boundary conditions, or equivalently, supercells: The system is treated as a periodic arrangement of $M \times M$ subsystems of size $L \times L$ each and overall periodic boundary conditions. Then, $M^{2}$ diagonalizations of a system with size $L^{2}$ are required, and the number of momentum points per direction is $M \times L$. We note that supercell calculations render the Hamiltonian matrix inevitably complex, while it is otherwise real in the collinear case considered here. Most results in the paper have been obtained for $L=64$ and $M=2$, and we show the transverse piece of $\chi^{\prime \prime}$ only.

We note that the present harmonic (or Gaussian) approximation for the problem with disorder is designed to capture disorder-induced mode broadening, but does not describe interaction-induced mode broadening, see also Sec. IIB below. Phase-space arguments imply that the former dominates at low energies whereas the latter can be expected to become important at elevated energies.

\section{Static structure factor}

The static spin structure factor is defined as

$$
S(\vec{q})=\frac{1}{2 N}\left[\sum_{i i^{\prime} m m^{\prime}}\left\langle\vec{S}_{i m} \cdot \vec{S}_{i^{\prime} m^{\prime}}\right\rangle e^{-i \vec{q} \cdot \vec{r}_{i i^{\prime} m m^{\prime}}}\right]_{\mathrm{av}}
$$

with $2 N$ the total number of spins and $\vec{r}_{i i^{\prime} m m^{\prime}}=\vec{r}_{i m}-$ $\vec{r}_{i^{\prime} m^{\prime}}$ the distance between a pair of spins. In the bilayer model, the ordered state displays a Bragg peak at $\vec{Q}=(\pi, \pi, \pi)$; for $N \rightarrow \infty$ its intensity is related to the staggered magnetization per spin via $M_{s}^{2}=S(\vec{Q}) /(2 N)$.

In Fig. 4 of the main text we show $S(\vec{q})$ for $q_{z}=\pi$; for the finite-size scaling $S(\vec{q})$ has been calculated in a meanfield approximation only, $\left\langle\vec{S}_{i m} \cdot \vec{S}_{i^{\prime} m^{\prime}}\right\rangle \rightarrow\left\langle\vec{S}_{i m}\right\rangle \cdot\left\langle\vec{S}_{i^{\prime} m^{\prime}}\right\rangle$. For small systems we have checked that the fluctuation corrections only lead to minor quantitative changes.

\section{BOND OPERATORS FOR THE CLEAN BILAYER MAGNET}

To make the presentation self-contained, we quickly summarize the most relevant results of the bond-operator

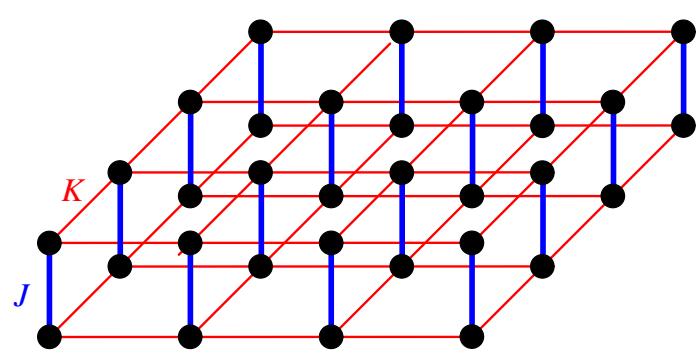

FIG. S1: Square-lattice bilayer Heisenberg model with interlayer coupling $J$ and intralayer coupling $K$.

approach to the bilayer Heisenberg magnet, Fig. S1, without quenched disorder.

\section{A. Harmonic theory: Non-interacting bosons}

A number of papers have applied versions of the bondoperator formalism to the bilayer Heisenberg model. In many cases, interactions between triplet excitations are neglected, i.e., only bilinear terms in the bond-operator Hamiltonian are kept. The approaches differ in the treatment of the Hilbert-space constraint: It is either fulfilled on average, leading to so-called bond-operator mean-field theory,,$\frac{1,3-5}{-}$ or ignored completely (at the quadratic level) in the spirit of linear spin-wave theory $\frac{6.7}{}$ Here we focus on Ref. 6 which follows the latter concept, as this allows one to consistently treat the ordered phase across the entire phase diagram such that Goldstone's theorem is automatically fulfilled. We also restrict ourselves to the case of zero applied field.

The basis transformation in Eq. (2) in the main text takes the following simple form:

$$
\tilde{t}_{i 0}^{\dagger}=\frac{t_{i 0}^{\dagger}+\lambda e^{i \vec{Q} \cdot \vec{R}_{i}} t_{i 3}^{\dagger}}{1+\lambda^{2}}, \quad \tilde{t}_{i 3}^{\dagger}=\frac{t_{i 3}^{\dagger}-\lambda e^{i \vec{Q} \cdot \vec{R}_{i}} t_{i 0}^{\dagger}}{1+\lambda^{2}}
$$

and $\tilde{t}_{i 1}^{\dagger}=t_{i 1}^{\dagger}, \tilde{t}_{i 2}^{\dagger}=t_{i 1}^{\dagger}$. Here $\lambda$ is parameterizes the linear combination of singlet and $z$ triplet, and $\vec{Q}=(\pi, \pi)$ is the ordering wavevector in the dimer lattice of the $\vec{R}_{i}$. Minimization of the product-state energy, $\mathcal{H}_{0}=\left\langle\tilde{\psi}_{0}|\mathcal{H}| \tilde{\psi}_{0}\right\rangle$, yields

$$
\lambda^{2}=\frac{4 K-J}{4 K+J} .
$$

For $K / J<1 / 4$ the system is paramagnetic, and from $\mathcal{H}_{2}$ one finds the energy dispersion to be

$$
\begin{aligned}
\omega_{\vec{q}} & =\sqrt{A_{\vec{q}}^{2}-B_{\vec{q}}^{2}}, \\
B_{\vec{q}} & =2 K \gamma_{\vec{q}}, A_{\vec{q}}=J+B_{\vec{q}}, \\
\gamma_{\vec{q}} & =\left(\cos q_{x}+\cos q_{y}\right) / 2,
\end{aligned}
$$

with a spin gap given by

$$
\Delta=\sqrt{J(J-4 K)}
$$


and a triplon bandwidth of

$$
W=\sqrt{J}(\sqrt{J+4 K}-\sqrt{J-4 K}) .
$$

For $K / J>1 / 4$ the system is in an antiferromagnetic state, with a product-state staggered magnetization

$$
M_{s}=\frac{\lambda}{1+\lambda^{2}}
$$

per spin. This value is somewhat reduced by Gaussian fluctuations; in particular in the limit of decoupled layers, $J \rightarrow 0$, where $\lambda=1$ one recovers the spin-wave value $M_{s}=0.66$ (Ref. $\underline{6}$ ).

\section{B. Beyond non-interacting bosons}

\section{Microscopic treatment}

Going beyond the non-interacting (i.e. linearized) theory requires to take into account the quartic interactions in $\mathcal{H}_{4}$ (cubic terms are absent from the bilayer model) as well as the hard-core constraint. An efficient approximation for the latter is given by the Brueckner theory, which yields an accurate value for the location of the critical point, as shown by Kotov et al! $?^{7}$ However, this approach cannot be easily generalized to the ordered phase. An earlier approach by Chubukov and Morr, $\underline{\underline{\underline{\beta}}}$ inspired by non-linear spin-wave theory, works in both phases, but suffers from divergencies at higher orders, probably because it lacks a small control parameter.

Notably, it can be shown that a systematic expansion can be constructed in the limit of large coordination number $z$ (or, alternatively, large spatial dimension $d$ ), i.e., using $1 / z$ (or $1 / d$ ) as a small parameter $\stackrel{9}{*}$ First, fluctuation corrections to the product state $\left|\tilde{\psi}_{0}\right\rangle$ vanish as $z \rightarrow \infty$; this is similar in spirit to spin-wave theory where fluctuation corrections vanish in the limit of large spin. Second, the harmonic fluctuations described by non-interacting triplons constitute the leading-order correction to the product state, i.e., their contribution to thermodynamic quantities is of order $1 / z$. Finally, higher-order corrections can be perturbatively calculated order by order in $1 / z$. The complexity is comparable

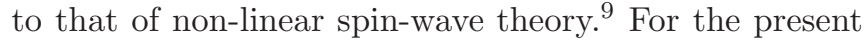
problem, with quenched disorder, corrections beyond the harmonic approximation can be calculated in principle, but are beyond the scope of this paper.

\section{Effective modelling}

In general, anharmonic effects contribute to thermodynamic quantities and modify spectral properties. If one restricts the attention to the dispersion and spectral weight of the triplon quasiparticles, then the effect of interactions may be captured in terms of renormalized model parameters, i.e., the full mode dispersion of the original model can be approximately represented as the dispersion of harmonic triplons in a renormalized model. This has been investigated in some detail for spin ladders in Ref. 10, where it was found that important spectral properties are well reproduced in such a simplied approach when compared against a numerical exact diagonalization of the original model.

For the QPT in the present bilayer model, this implies that the interaction-induced shift of the critical point from $(J / K)_{c}=4$ to $(J / K)_{c}=2.5220(1)$ (Ref. 11) - can be captured, to leading order, in terms of renormalized $J$, $K$. In this paper, we account for this fact in the simplest fashion, namely by specifying the parameter ratio $J / K$ relative to the QCP location.

\section{RESULTS FOR DIFFERENT TYPES OF DISORDER}

In the main paper, results were discussed for disordered interlayer (or intra-dimer) couplings $J$, specifically a small concentration of weakened (Figs. 1-4) or strengthened (Fig. 5) interlayer couplings. Here we first discuss some general aspects of disorder, also with an eye towards real materials, and then present data for other types of disorder.

\section{A. Griffiths regime and paramagnetic gap}

For the bounded disorder distributions considered in this paper, there is always a well-defined (hard) excitation gap for sufficiently small $K / J$. Upon increasing $K$, the gap closes at the boundary to the quantum Griffiths regime 12 In our mean-field-based treatment, (weak) magnetic order sets in at this point; non-linear fluctuation corrections would be required to obtain the correct physical behavior, namely a magnetic phase transition inside the Griffiths regime. In other words, at the level of our approximation a paramagnetic quantum Griffiths regime is absent, and the entire Griffiths regime is magnetically ordered. Despite this, we can expect that the properties of the excitations are captured correctly except at ultra-low energies.

Depending on the type of disorder, the "apparent" energy gap in $\chi^{\prime \prime}$ may be much larger than the actual gap, namely if the intensity in $\chi^{\prime \prime}$ is very small for small $\omega$ - this happens if the low-energy excitations are carried by rare configurations (i.e. rare clusters of small $J$ or large $K$ ). This effect can be seen in Fig. 2a of the main paper, where a true gap is absent at $p=0.05$, but the susceptibility has an apparent gap of $\approx 0.1 \mathrm{~J}$. 
(a) $K_{1} / K_{\mathrm{c}}=0.75, p=0.02$

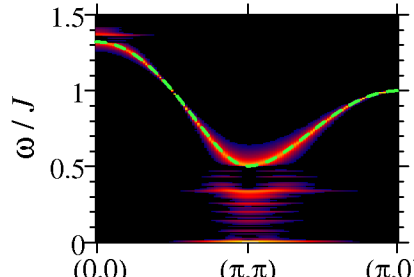

$(0,0)$

$(\pi, \pi)$ (b) $K_{1} / K_{\mathrm{c}}=0.83, p=0.02$

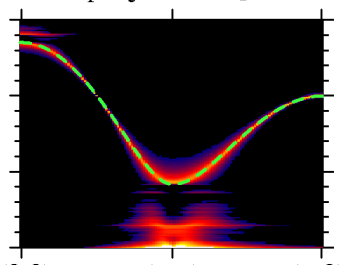

$(\pi, \pi)$ (c) $K_{1} / K_{\mathrm{c}}=0.92, p=0.02$

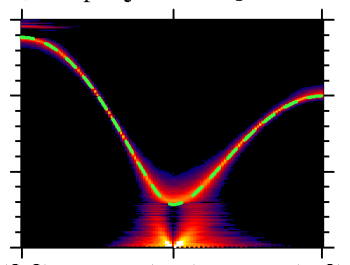

$(\pi, \pi)$ $(\pi, 0)(0,0)$

FIG. S2: Susceptibility $\chi^{\prime \prime}(\vec{q}, \omega)$, but now for disorder in the intralayer couplings $K$ : a density of $p$ dimers has strong intralayer couplings $K_{2}=2 K_{1}$ with all neighbors.

(a) $K_{1} / K_{\mathrm{c}}=0.96, p=0.2$

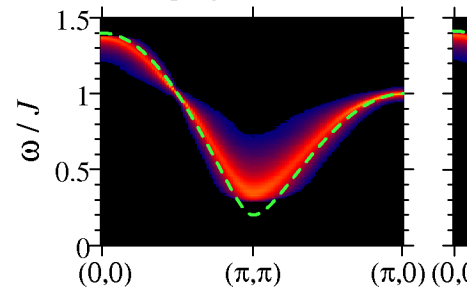

(b) $K_{\mathrm{l}} / K_{\mathrm{c}}=1.01, p=0.2$

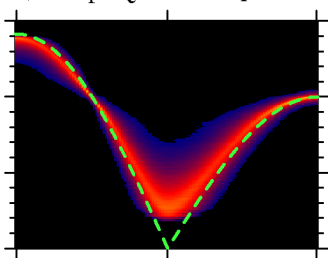

$(\pi, \pi)$ (c) $K_{1} / K_{\mathrm{c}}=1.07, p=0.2$

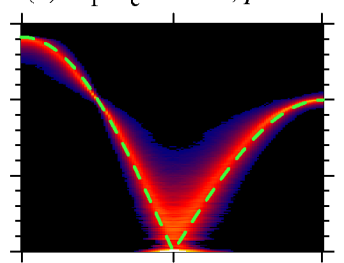

$(\pi, \pi)$

$(\pi, 0)(0,0)$

(d) $K_{1} / K_{\mathrm{c}}=1.12, p=0.2$

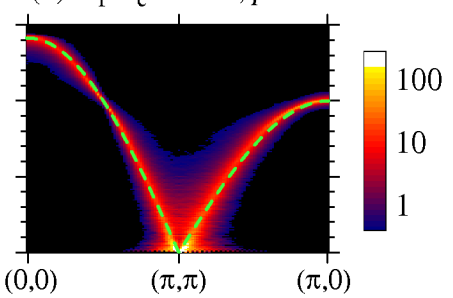

FIG. S3: Susceptibility $\chi^{\prime \prime}(\vec{q}, \omega)$ for disordered intralayer couplings $K$ as in Fig. S2 but now a density of $p$ dimers has weak intralayer couplings with $K_{2}=K_{1} / 2$ with all neighbors.

\section{B. Disorder in experiments}

Apart from fundamental interest, specific models of disorder may be motivated by the experimental situation. Here, bond disorder is introduced via ligand substitution, as e.g. in $\mathrm{Tl}_{1-x} \mathrm{~K}_{x} \mathrm{CuCl}_{3}, \stackrel{13,14}{ }\left(\mathrm{C}_{4} \mathrm{H}_{12} \mathrm{~N}_{2}\right) \mathrm{Cu}_{2}\left(\mathrm{Cl}_{1-x} \mathrm{Br}_{x}\right)_{6}, \stackrel{15,16}{, 19}$

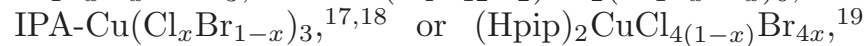
such that bimodal disorder models appear appropriate.

In general, such substitutions influence both intradimer $(J)$ and inter-dimer $(K)$ couplings; this can be deduced from the end members of the substitution series. For instance, $\mathrm{TlCuCl}_{3}$ and $\mathrm{KCuCl}_{3}$ have drastically different spins gaps of 0.7 and $2.5 \mathrm{meV}$, respectively, and fits to INS data results in values of $J=$ $5.42 \mathrm{meV}$ and $K_{1,2,3}=(-0.47,-1.43,0.62) \mathrm{meV}$ for $\mathrm{TlCuCl}_{3}$, in contrast to $J=4.29 \mathrm{meV}$ and $K_{1,2,3}=$ $(-0.21,-0.34,0.37) \mathrm{meV}$ for $\mathrm{KCuCl}_{3}$; here $K_{1,2,3}$ are the strongest couplings in the three-dimensional dimer network of these materials $\stackrel{20}{2}$ Therefore, a substitution $\mathrm{Tl} \rightarrow \mathrm{K}$ in $\mathrm{TlCuCl}_{3}$ tends to increase the spin gap; the same applies to $\mathrm{Cl} \rightarrow \mathrm{Br}$ in $\left(\mathrm{C}_{4} \mathrm{H}_{12} \mathrm{~N}_{2}\right) \mathrm{Cu}_{2} \mathrm{Cl}_{6}$ and (Hpip) ${ }_{2} \mathrm{CuCl}_{4}$.

It can be expected that partial substitution of ligands locally changes $J$ and/or $K$ couplings, but this happens in a correlated fashion: A single substituted ligand atom influences numerous local bond lengths and angles, such that various couplings in the vicinity of the substituent are modified - this applies in particular to interdimer couplings which typically result from multiple exchange paths. Therefore, models of uncorrelated bimodal disorder are a strong simplification and cannot fully capture the disorder physics of real materials. We have therefore, in addition to bimodal uncorrelated disorder in $J$, also considered correlated disorder in $K$, see below.

\section{Bimodal bond disorder}

The case of disordered interlayer couplings $J$ was extensively discussed in the main text. In Figs. 1-4 the focus was on a small concentration of weakened interlayer couplings. Those have the tendency to reduce the spin gap by inducing in-gap states, which are strongly localized and lead to broad modes at lowest energies near the transition (Fig. 1).

The opposite case of a small concentration $p$ of stronger interlayer $(J)$ couplings was shown in Fig. 5 of the main paper. As a consequence of a few strong $J$ bonds, the finite- $p$ excitation spectrum now shows a larger apparent gap as compared to the $p=0$ case, and ordering tendencies are suppressed. The few strong bonds induce non-dispersing excitations above the main band and with weak intensity; at the same time the overall width of the main band is reduced. Near the transition, Fig. 5(c) there is still significant mode broadening at low energies.

We now turn to disorder in the intralayer (or interdimer) couplings $K$. For uncorrelated disorder in $K$, we have found that disorder effects are much weaker than that of uncorrelated disorder in $J$, i.e., uncorrelated $K$ disorder efficiently averages out because of the presence of many (here eight) $K$ bonds for each dimer. Considering that uncorrelated disorder is unrealistic for real materials (Sec. IIIB), we have then chosen to model correlated 
(a) $K / K_{\mathrm{c}}=0.88$

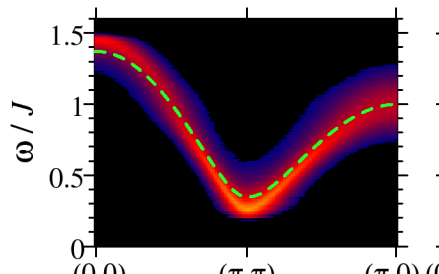

$(0,0)$

$(\pi, \pi)$ (b) $K / K_{\mathrm{c}}=0.92$

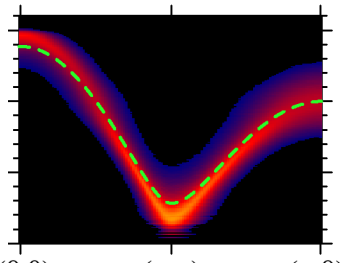

$(\pi, \pi)$ (c) $K / K_{\mathrm{c}}=0.94$

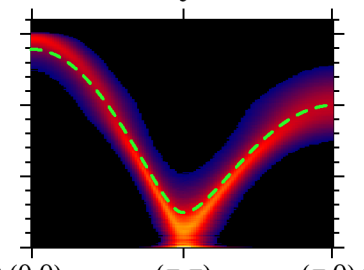

$(\pi, \pi)$ $(\pi, 0)(0,0)$ (d) $K / K_{\mathrm{c}}=0.96$

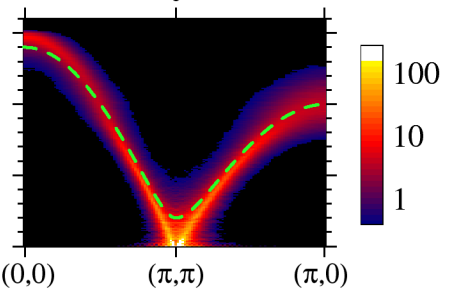

FIG. S4: Susceptibility $\chi^{\prime \prime}(\vec{q}, \omega)$, but now for a box distribution of interlayer couplings $J$ with a width $\delta J=J / 2$, i.e., the $J_{i}$ in Eq. (1) vary in the interval $[0.75 J, 1.25 J]$.

(a) $K / K_{\mathrm{c}}=0.88$

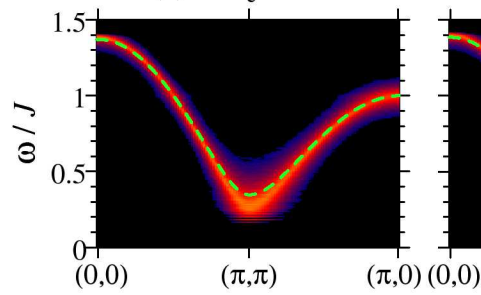

(b) $K / K_{\mathrm{c}}=0.92$

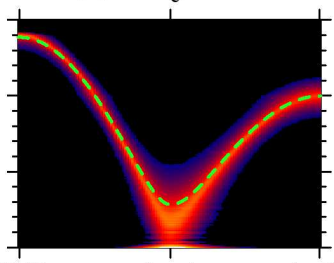

(c) $K / K_{\mathrm{c}}=0.94$

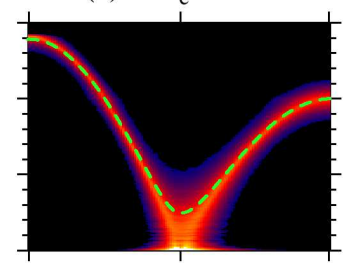

(d) $K / K_{\mathrm{c}}=0.96$

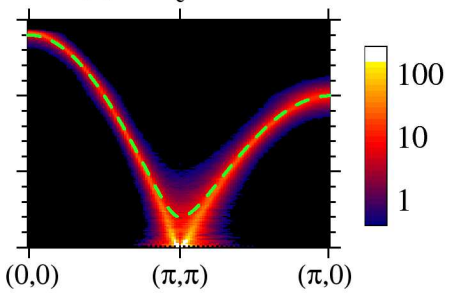

FIG. S5: Susceptibility $\chi^{\prime \prime}(\vec{q}, \omega)$, but now for a box distribution of intralayer couplings $K$ with a width $\delta K=K$, i.e., the $K_{i i^{\prime}}^{m m}$ in Eq. (1) vary in the interval $[0.5 K, 1.5 K]$.

disorder in the following fashion: We randomly choose a (small) fraction $p$ of dimers, mimicking the location of dopants, and then give all $K$ bonds emerging from such dimers the strength $K_{2}$, while the remaining $K$ bonds have strength $K_{1}$. Results are shown in Figs. S2 and S3 for the cases of $p$ dimers with stronger and weaker intralayer couplings, respectively.

Clearly, a small concentration of sites with stronger intralayer couplings, Fig. S2, has an effect similar to a small concentration of weaker interlayer couplings, Fig. 1: The dopants introducde significant in-gap spectral weight and drives the system towards the ordered state. The in-gap states themselves now show significant energetic structure, arising from cluster of two, three, and more dimers sites with stronger intradimer coupling.

In contrast, a small concentration of sites with weaker intralayer couplings, Fig. S3. leads to spectra similar to the case of a small concentration of stronger interlayer couplings, Fig. 5 of the main paper, with bandwidth reduction and mode broadening being the dominant effects.

\section{Box bond disorder}

For completeness, we have also considered uncorrelated continuous distributions of coupling constants. Specifically, we have studied box distributions for either $J$ (with non-disordered $K$ ) or $K$ (with non-disordered $J$ ). Corresponding results are shown in Figs. S4 and S5. respectively.

Not surprisingly, box distributions tend to enlarge the effective bandwidth of magnetic excitations, simply be- cause the range of available couplings increases: For instance, a variation of $J$ tends to shift the center energy of the triplon band, such that a system with box-distributed $J$, Fig. S4 effectively samples bands corresponding to a range of $J$ values. Similarly, a variation of $K$ tends to modify the bandwidth, such that the bandwidth for boxdistributed $K \in[K-\delta K / 2, K+\delta K / 2]$ is larger than that corresponding to the pure- $K$ case. However, the increase in bandwidth is moderate even for large $\delta K$, Fig. S5. which demonstrates that uncorrelated disorder in the intradimer couplings averages out as noted above.

Smearing of the excitations (but no splitting) is visible at all energies for both box distributions, with smearing again being most pronounced at low energies near the transition. In particular, strongly broadened low-energy modes are visible in Figs. \$4

\section{EVOLUTION OF MODE BROADENING AND BAND WIDTH}

The calculated $\chi^{\prime \prime}(\vec{q}, \omega)$ enables a quantitative analysis of the evolution of spectral properties with the disorder level, in particular the energetic mode broadening and the triplon bandwidth.

A first impression can be obtained by considering the energy-dependent response at fixed wavevector, as shown in Fig. S6 (a,b) for the putative ordering wavevector $\vec{q}=\vec{Q}=(\pi, \pi)$. Both panels are for different disorder in the interlayer couplings: (a) has a small concentration of strong interlayer bonds, which have the simple tendency to broaden the mode and shift it to higher energies. 
In contrast, (b) has a small concentration of weak interlayer bonds, which have two effects: The "main" mode is slightly shifted upwards, but a significant amount of weight is created inside the gap.

In the case (a) of few strong interlayer bonds, it makes sense to quantify the mode broadening. This is done in Fig. S66(c) for $\vec{q}=(\pi, \pi)$ and two different values of $K / K_{c}$ corresponding to the data depicted in Fig. $5(\mathrm{a}, \mathrm{b})$ of the main paper. The data are consistent with a linewidth scaling linearly with $p$, with a prefactor which increases upon approaching the QPT. (As noted before, the broadening is largest in the regime where the apparent spin gap closes, see Fig. 5(c).)

Fig. S6 (d) displays the evolution of the magnon bandwidth for the same case of few strong interlayer bonds; the small-intensity non-dispersing states at the upper end of the spectrum, Fig. 5, have been ignored in this analysis. The bandwidth decreases as expected from Eq. (S19); the decrease is approximately linear for small $p$. This qualitatively matches recent experimental INS data. $\underline{16,18}$

We finally note that non-linearities, i.e., triplon-triplon interactions, will also lead to broadening and renormalization effects, which are not part of the present calculation. However, interaction-induced broadening is typically small at low energies, i.e., near the bottom of the excitation band, because of phase-space restrictions for particle decay. Therefore, the disorder effects captured by our calculations can be expected to dominate at low energies.
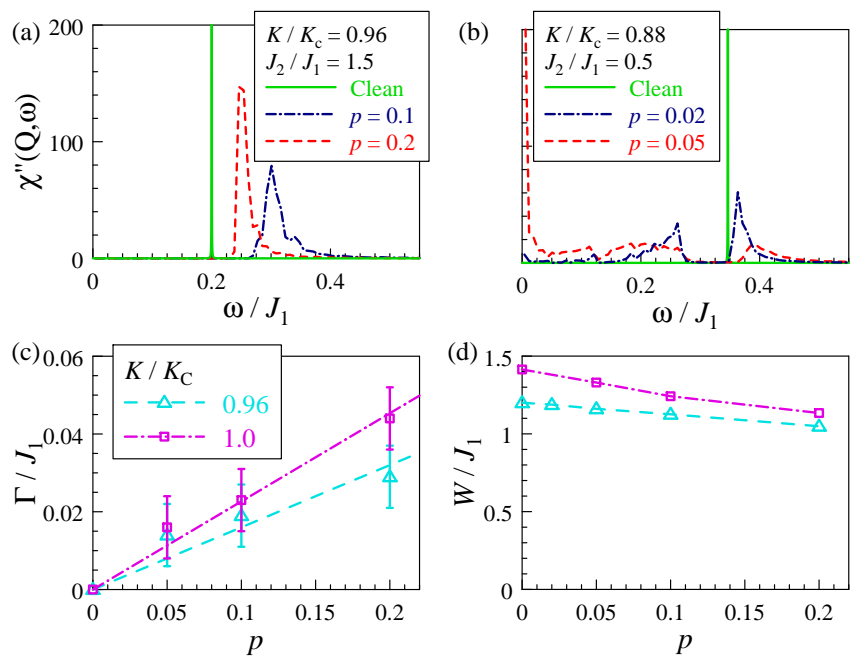

FIG. S6: (a) Excitation spectrum $\chi^{\prime \prime}(\vec{q}, \omega)$ at fixed $\vec{q}=\vec{Q}=$ $(\pi, \pi)$, for a small concentration of strong interlayer bonds with $J_{2}=3 J_{1} / 2$, as in Fig. 5 of the main paper. (b) Same as (a), but for a small concentration of weak interlayer bonds with $J_{2}=J_{1} / 2$, as in Fig. 1 of the main paper. (c,d) Linewidth at $\vec{Q}=(\pi, \pi)$ and triplon bandwidth for the case of of strong interlayer bonds with $J_{2}=3 J_{1} / 2$ of function of $p$. The error bars arise primarily from the limited energy resolution of our finite-size calculation; the lines are guide to the eye.

\section{LOCALIZATION OF LOW-ENERGY MODES}

As illustrated in Fig. 3(c,d) of the main text, the low-energy excitation modes of the disordered coupleddimer magnet tend to be spatially localized. This can be quantified by considering the inverse participation ratio (IPR) of the eigenmodes, with the general definition of $\mathrm{IPR}=\sum_{i}\left|\psi_{i}\right|^{4}$ for a wavefunction $|\psi\rangle$, and $\psi_{i}=\langle i \mid \psi\rangle$ where $i$ is a lattice site. The finite-size behavior of the IPR is indicative of localization: For spatially extended states, the IPR scales with the system size as $1 / N$, whereas it approaches a constant for localized states. For exponential localization, the IPR value is then a measure of the localization length, IPR $\propto 1 / \xi^{d}$.

For the eigenvectors $\tau_{n}$ of the bosonic Bogoliubov transformation (S10) we define the IPR as

$$
\operatorname{IPR}_{n}=\sum_{i \alpha}\left(\left|u_{n i \alpha}\right|^{2}-\left|v_{n i \alpha}\right|^{2}\right)^{2},
$$

note that $\sum_{i \alpha}\left(\left|u_{n i \alpha}\right|^{2}-\left|v_{n i \alpha}\right|^{2}\right)=1$. Some previous papers ${ }^{2,21}$ have employed an alternative definition,

$$
\operatorname{IPR}_{n}^{\prime}=\frac{\sum_{i \alpha}\left|v_{n i \alpha}\right|^{4}}{\sum_{i \alpha}\left|v_{n i \alpha}\right|^{2}}
$$

we have checked that both quantities show qualitatively similar properties.

Energy-resolved histograms of IPR values (S21) are shown in Fig. $\mathrm{S} 7$ for the same parameters as employed in Figs. 1(g), 3, 4 of the main text, i.e., $p=0.02$ weak interlayer bonds. A scaling with inverse system size is visible for essentially all energies $\omega>0.35 J$, indicating extended states, whereas states with $\omega<0.3 J$ are clearly localized. Considering that the spin gap of the clean system is $\Delta=0.28 \mathrm{~J}$, it is obvious that it is the disorder-induced states below the gap which are subject to localization. (The somewhat enhanced IPR at both $\omega / J \approx 1$ and the

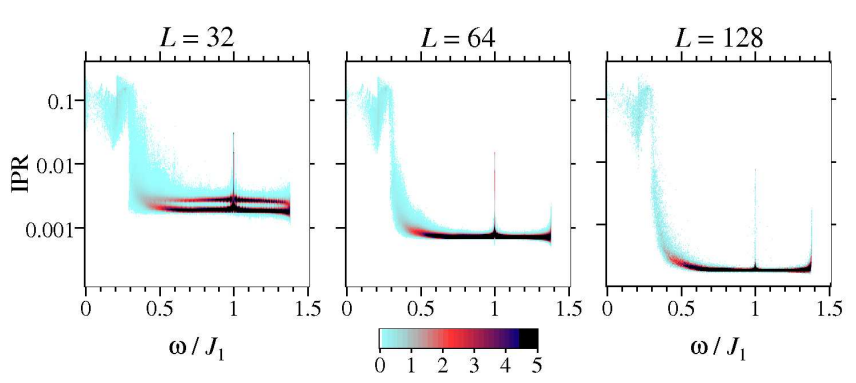

FIG. S7: Inverse participation ratio (S21) for the eigenmodes of $\mathcal{H}_{2}$ of the disordered bilayer Heisenberg model, with $K / K_{c}=0.92$ and $p=0.02$ weak interlayer bonds with $J_{2}=J_{1} / 2$ as in Fig. $1(\mathrm{~g})$ of the main paper. The panels show the IPR as function of the mode energy for different system sizes $L \times L$; the color encodes the average number of modes (per IPR and energy interval and per disorder realization) in a histogram fashion. Localization tendencies, with the IPR being independent of system size, are pronounced for energies $\omega<0.3 J$, i.e., below the gap of the clean system. 

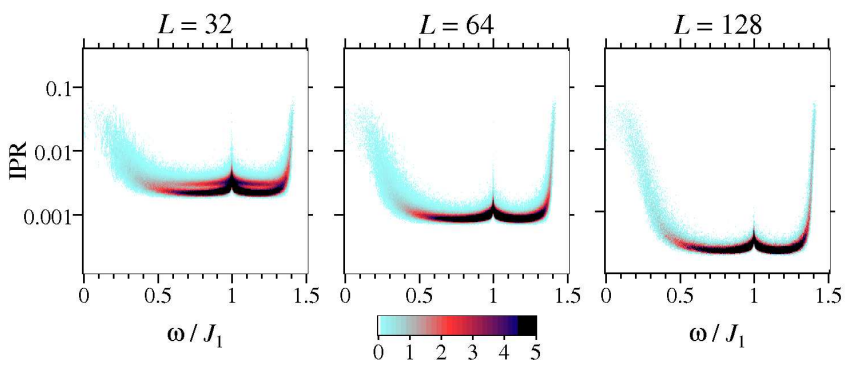

FIG. S8: Same as Fig. S7 but for a box distribution of intralayer couplings $K$ with $K / K_{c}=0.92$ as in Fig. S5 (b).

upper band edge arises primarily from the flat pieces of the mode dispersion.) Comparing Figs. 1(g) and \$ 57 also shows that energies with a large IPR correlate with those showing a large broadening in momentum space, both indicative of a short real-space localization length.

Fig. S8 illustrates similar localization tendencies for a box distribution of couplings, albeit with a somewhat larger localization length. Here, localization takes place a both band edges, but concerns a broader energy range near the band bottom as compared to the top.

We conclude that disorder-induced localization of lowenergy modes is generic near the QPT of the bilayer Heisenberg model.

\section{RELATION TO BROADENING AND LOCALIZATION PHENOMENA IN PREVIOUS WORK ON DISORDERED MAGNETS}

The physics of defects in magnetic insulators is an intensely studied subject. It has long been recognized that defects modify the spectrum of magnetic excitations which can be probed in optical, NMR, or neutronscattering experiments $\stackrel{22}{2}$ Early works mainly dealt with ordered magnets where, via chemical substitution, impurities with a spin different from that of the host material were introduced $\underline{22-24}$ This includes the particularly simple case of spinless impurities, a situation which has seen intense theoretical modelling over the last decade $\underline{21,25,26}$ In addition, spinless impurities in quantum-disordered paramagnets were also studied $\stackrel{27,28}{\underline{2}}$

The present work differs in two main aspects from previous investigations of spectral properties of disordered magnets: First, we concentrate on systems in the vicinity of a magnetic quantum critical point, as opposed to systems inside stable phases (either ordered or disordered). Quantum critical systems are inherently unstable and thus more susceptible to disorder. Second, we study a rather weak form of disorder, namely random bonds or, technically speaking, random-mass disorder. Most earlier works instead dealt with missing or substituted spins which induce random Berry phases. The latter form of disorder is much stronger than random-mass disorder: for instance, any small amount of spinless impuri- ties brought into a confined gapped quantum paramagnet induces zero-temperature magnetic order, $\stackrel{29-33}{\underline{2}}$ whereas weak random-mass disorder leaves a gapped paramagnetic phase intact.

In terms of results for the excitation spectrum, $\chi^{\prime \prime}(\vec{q}, \omega)$, these differences are visible in various respects: (i) In our calculations, disorder effects are strongest near quantum criticality - a regime not covered by earlier work. In contrast, in ordered phases with Goldstone modes, disorder tends to decouple from the excitations in the low-energy limit 34 this sharpening of Goldstone modes is also seen in our results, see e.g. Figs. 1(e), S2(d), S3(d), S4(d), and S5(d). (ii) Near criticality, we observe a large broadening already at small impurity concentrations of $2 \%$ (despite the fact that we consider random-mass disorder only). In most previous work comparably large effects were present only for defect concentrations of $10 \%$ and above; notable exceptions are spinless impurities in certain spin-gap magnets 28 and also in cuprates 35 (with the latter being close to a magnetic QPT).

Paranthetically, I note that a detailed analysis of lineshapes has not been performed here (and is also missing from most previous works) - this is left for future studies.

Finally, the aspect of spatial localization of the excitation modes deserves a discussion. There is consensus that sufficiently strong disorder will induce mode localization, and that weak disorder will not localize modes in high dimensions $\stackrel{36}{\underline{3}}$ However, localization properties of magnetic excitations in low dimensions are not fully understood: While the standard arguments for Anderson localization predict that all single-particle states are localized in generic non-interacting $2 \mathrm{~d}$ disordered systems, the following ingredients make the problem nontrivial: (i) Magnons as well as triplons are hard-core bosons, i.e., their interactions are not negligible. Understanding of localization properties of bosons in the presence of hard-core interactions is an unsolved problem. (ii) Even if interactions are neglected, the underlying (magnon or triplon) single-particle Hamiltonian has a bosonic Bogoliubov-de-Gennes structure, such that the standard arguments for localization do not directly apply. In addition, one has to distinguish systems with and without Goldstone modes $\underline{\underline{34}}$ Indeed, it has been argued for certain $2 \mathrm{~d}$ random magnets that delocalized spin-wave modes can exist, which are energetically separated from localized modes by a mobility edge $\underline{37}$ Taking into account (i), this has to be considered speculative at present.

In diluted magnets near the percolation threshold, excitations at elevated energies have been discussed in terms of fractons, i.e., localized modes which live on a percolating network of sites $\underline{38,39}$ This physics is not relevant for the cases of (weak) bond disorder studied here, but it will be extremely interesting to consider excitations subject to the interplay of both percolation and quantum criticality, as e.g. in the diluted bilayer model studied in Ref. 40 . 
1 S. Sachdev and R. N. Bhatt, Phys. Rev. B 41, 9323 (1990).

2 S. Wessel and I. Milat, Phys. Rev. B 71, 104427 (2005).

${ }^{3}$ Y. Matsushita, M. P. Gelfand, and C. Ishii, J. Phys. Soc. Jpn. 68, 247 (1999).

4 D.-K. Yu, Q. Gu, H.-T. Wang, and J.-L. Shen, Phys. Rev. B 59, 111 (1999).

5 R. Ganesh, S. V. Isakov, and A. Paramekanti, Phys. Rev. B 84, 214412 (2011).

6 T. Sommer, M. Vojta, and K. W. Becker, Eur. Phys. J. B 23, 329 (2001).

7 V. N. Kotov, O. P. Sushkov, Zheng Weihong, and J. Oitmaa, Phys. Rev. Lett. 80, 5790 (1998).

8 A. V. Chubukov and D. K. Morr, Phys. Rev. B 52, 3521 (1995).

${ }^{9}$ D. Joshi and M. Vojta, in preparation.

${ }^{10}$ R. Eder, Phys. Rev. B 57, 12832 (1998).

11 L. Wang, K. S. D. Beach, and A. W. Sandvik, Phys. Rev. B 73, 014431 (2006).

12 T. Vojta, J. Phys. A 39, R143 (2006), and references therein.

13 A. Oosawa and H. Tanaka, Phys. Rev. B 65, 184437 (2002).

14 F. Yamada, H. Tanaka, T. Ono, and H. Nojiri, Phys. Rev. B 83, 020409(R) (2011).

15 D. Hüvonen, S. Zhao, M. Månsson, T. Yankova, E. Ressouche, C. Niedermayer, M. Laver, S. N. Gvasaliya, and A. Zheludev, Phys. Rev. B 85, 100410 (2012).

16 D. Hüvonen, S. Zhao, G. Ehlers, M. Månsson, S. N. Gvasaliya, and A. Zheludev, Phys. Rev. B 86, 214408 (2012).

17 H. Manaka, A. V. Kolomoiets, and T. Goto, Phys. Rev. Lett. 101, 077204 (2008).

18 B. Nafradi, T. Keller, H. Manaka, U. Stuhr, A. Zheludev, and B. Keimer, Phys. Rev. B 87, 020408(R) (2013).

19 S. Ward, P. Bouillot, H. Ryll, K. Kiefer, K. W. Krämer, C. Rüegg, C. Kollath, and T. Giamarchi, J. Phys. Condens. Matter 25, 014004 (2013).

20 N. Cavadini, G. Heigold, W. Henggeler, A. Furrer, H.-U. Güdel, K. Krämer, and H. Mutka, Phys. Rev. B 63, 172414 (2001).

21 E. R. Mucciolo, A. H. Castro Neto, and C. Chamon, Phys. Rev. B 69, 214424 (2004).

${ }^{22}$ R. A. Cowley and W. J. L. Buyers, Rev. Mod. Phys. 44,
406 (1972).

23 S. E. Nagler, W. J. L. Buyers, R. L. Armstrong, and R. A. Ritchie, J. Phys. C 17, 4819 (1984).

24 Y. J. Uemura and R. J. Birgeneau, Phys. Rev. B 36, 7024 (1987).

25 A. L. Chernyshev, Y. C. Chen, and A. H. Castro-Neto, Phys. Rev. B 65, 104407 (2002).

26 A. Chakraborty and G. Bouzerar, Phys. Rev. B 81, 172406 (2010).

27 G. Xu, G. Aeppli, M. E. Bisher, C. Broholm, J. F. DiTusa, C. D. Frost, T. Ito, K. Oka, R. L. Paul, H. Takagi, and M. M. J. Treacy, Science 289, 419 (2000).

28 S. Haravifard, S. R. Dunsiger, S. El Shawish, B. D. Gaulin, H. A. Dabkowska, M. T. F. Telling, T. G. Perring, and J. Bonca, Phys. Rev. Lett. 97, 247206 (2006).

29 M. Imada and Y. Iino, J. Phys. Soc. Jpn. 66, 568 (1997).

30 C. Yasuda, S. Todo, M. Matsumoto, and H. Takayama, Phys. Rev. B 64, 092405 (2001).

31 S. Wessel, B. Normand, M. Sigrist, and S. Haas, Phys. Rev. Lett. 86, 1086 (2001).

32 M. Hase et al., Phys. Rev. Lett. 71, 4059 (1993).

33 A. Oosawa, T. Ono, and H. Tanaka, Phys. Rev. B 66, 020405(R) (2002).

34 V. Gurarie and J. T. Chalker, Phys. Rev. B 68, 134207 (2003).

35 H. F. Fong, P. Bourges, Y. Sidis, L. P. Regnault, J. Bossy, A. Ivanov, D. L. Milius, I. A. Aksay, and B. Keimer, Phys. Rev. Lett. 82, 1939 (1999); A. Suchaneck, V. Hinkov, D. Haug, L. Schulz, C. Bernhard, A. Ivanov, K. Hradil, C. T. Lin, P. Bourges, B. Keimer, and Y. Sidis, Phys. Rev. Lett. 105, 037207 (2010).

36 P. B. Weichman, Mod. Phys. Lett. B 22, 2623 (2008).

37 J. P. A. Zuniga and N. Laflorencie, preprint arXiv:1304.7636

38 T. Nakayama, K. Yakubo, and R. L. Orbach, Rev. Mod. Phys. 66, 381 (1994).

39 H. Ikeda, J. A. Fernandez-Baca, R. M. Nicklow, M. Takahashi, and K. Iwasa, J. Phys. Cond. Matter 6, 10543 (1994).

40 A. W. Sandvik, Phys. Rev. Lett. 96, 207201 (2006). 